\title{
Inhibition of Plant-Pathogenic Fungi by the Barley Cystatin Hv-CPI (Gene Icy) Is Not Associated with Its Cysteine-Proteinase Inhibitory Properties
}

\author{
M. Martínez, E. López-Solanilla, P. Rodríguez-Palenzuela, P. Carbonero, and I. Díaz \\ Laboratorio de Bioquímica y Biología Molecular, Departamento de Biotecnología-UPM, ETSI Agrónomos, 28040 Madrid, \\ Spain.
}

Submitted 8 May 2003. Accepted 13 June 2003.

\begin{abstract}
The recombinant barley cystatin Hv-CPI inhibited the growth of three phytopathogenic fungi (Botrytis cinerea, Colletotrichum graminicola, and Plectosphaerella cucumerina) and the saprotrophic fungus Trichoderma viride. Several mutants of barley cystatin were generated by polymerase chain reaction approaches and both their antifungal and their cysteine-proteinase inhibitory properties investigated. Point mutants $R^{38} \rightarrow G, Q^{63} \rightarrow L$, and $Q^{63} \rightarrow P$ diminished their capacity for inhibiting papain and cathepsin B, retaining their antifungal properties. However, mutant $C^{68} \rightarrow G$ was more active for papain and cathepsin $B$ than the wild type. These results indicate that in addition to the consensus cystatin-reactive site, $Q^{63}-V^{64}-V^{65}-A^{66}-G^{67}$, the $A^{37}-R^{38}-F^{39}-A^{40}-V^{41}$ region, common to all cereal cystatins, and the $C^{68}$ residue are important for barley cystatin activity. On the other hand, the $K^{92} \rightarrow P$ mutant is inactive as a fungicide, but still retains measurable inhibitory activity for papain and cathepsin B. Against $B$. cinerea, the antifungal effect of $\mathrm{Hv}-\mathrm{CPI}$ and of its derived mutants does not always correlate with their activities as proteinase inhibitors, because the $Q^{63} \rightarrow P$ mutant is inactive as a cystatin, while still inhibiting fungal growth, and the $K^{92} \rightarrow P$ mutant shows the reciprocal effects. These data indicate that inhibition of plant-pathogenic fungi by barley cystatin is not associated with its cysteine-proteinase inhibitory activity. Moreover, these results are corroborated by the absence of inhibition of intra- and extramycelia-proteinase activities by barley cystatin and by other well-known inhibitors of cysteine-proteinase activity in the fungal zymograms of B. cinerea.
\end{abstract}

Additional keywords: antifungal activity, site-directed mutagenesis.

Cystatins are cysteine-protease inhibitors, widely distributed in vertebrates, invertebrates, and plants. Those from plants, referred to as phytocystatins (PhyCys), comprise more than 60 members (Bateman et al. 2002), which cluster in a major evolutionary tree branch of the cystatin superfamily of proteins (Margis et al. 1998). In addition to the reactive site motif QXVXG and the $\mathrm{W}$ residue located at the $\mathrm{C}$-terminal part of

Corresponding author: I. Diaz; Telephone: 34-91-3365705; Fax: 34-913365757; E-mail: Isabel@bit.etsia.upm.es. the protein (Arai et al. 1991), common to all plant and animal cystatins, the PhyCys from cereals are characterized by a molecular mass in the range of 12 to $16 \mathrm{kDa}$, the absence of disulphide bonds, and the presence of a consensus ARFAV sequence in the N-terminal half of the protein. Several cystatin isoforms and cystatin-encoding genes, with different spatial and temporal expression patterns and with different inhibitory activities towards cysteine-proteinases, have been described in maize, wheat, and rice (Abe et al. 1996; Corre-Menguy et al. 2002; Kondo et al. 1990). The two main functions that have been assigned to PhyCys are i) the regulation of endogenous protein turnover and ii) plant defense. The defense role is supported by the inhibitory activity toward digestive proteinases from insect pests and nematodes (Kuroda et al. 1996; Pernas et al. 1998; Siqueira-Junior et al. 2002; Zhao et al. 1996), together with their inducible expression by wounding and methyl jasmonate (Pernas et al. 2000a; Siqueira-Junior et al. 2002; Zhao et al. 1996). The protective function also has been supported by the enhanced resistance obtained against insects, nematodes, slugs, and potyviruses in transgenic plants overexpressing PhyCys genes (Delledonne et al. 2001; Gutierrez-Campos et al. 1999; Vain et al. 1998; Walker et al. 1999). Moreover, antifungal and antimite activities have been described for certain PhyCys (Joshi et al. 1998; Pernas et al. 1999, 2000b; Siqueira-Junior et al. 2002; 1999; Soares-Costa et al. 2002).

The best-studied PhyCys to date is the oryzacystatin I (OCI) from rice seed, whose three-dimensional structure recently was determined by ${ }^{1} \mathrm{H}$ and ${ }^{15} \mathrm{~N}$ NMR spectroscopy (Nagata et al. 2000). The main body ( $\mathrm{E}^{13}$ to $\mathrm{D}^{97}$ ) of the 102 amino acid residues of OC-I consists of an $\alpha$-helix, where the ARFAV motif is located, and of five antiparallel $\beta$-sheets $\left(\beta_{1} \beta_{2} \beta_{3} \beta_{4} \beta_{5}\right)$. The $\beta_{1}$ sheet is situated near the $\mathrm{N}$-terminal of the $\alpha$-helix, while the remaining $\beta$ sheets are located close to the $C$ terminal part of the molecule. At the end of $\beta_{2}$, in the hairpin loop connecting it to the $\beta_{3}$ sheet, the reactive site QVVAG is situated.

We previously have reported (Gaddour et al. 2001) the cloning and molecular characterization of a cDNA from developing barley endosperm encoding the cystatin Hv-CPI (gene Icy) of $11.8 \mathrm{kDa}$, which is highly related in sequence to the rice OC-I; and, although is ubiquitously expressed, its expression in leaves is induced by low temperatures and anaerobiosis. The recombinant Hv-CPI protein expressed in Escherichia coli was efficient in inhibiting papain, ficin, and chymopapain in vitro, whereas it was inactive against bromelain (Gaddour et al. 2001). In this article, we demonstrate 
that the barley Hv-CPI also has antifungal properties and show, using site-directed mutagenesis, that mutation of the reactive site does not affect its antifungal properties. These data, together with the lack of intra- or extracellular cysteineproteinase activities in $B$. cinerea, in zymogram conditions where serine-proteinases could be detected, suggest that cysteine-protease inhibition is not an important factor in determining the antifungal properties of barley cystatin. Sitedirected mutagenesis also has provided an improved Hv-CPI, mutant $\mathrm{C}^{68} \rightarrow \mathrm{G}$ with inhibition constants (Kis) toward papain and cathepsin B, one and two orders of magnitude lower than the wild type, respectively. These properties will be interesting for the use of mutants in transgenic crops in the future.
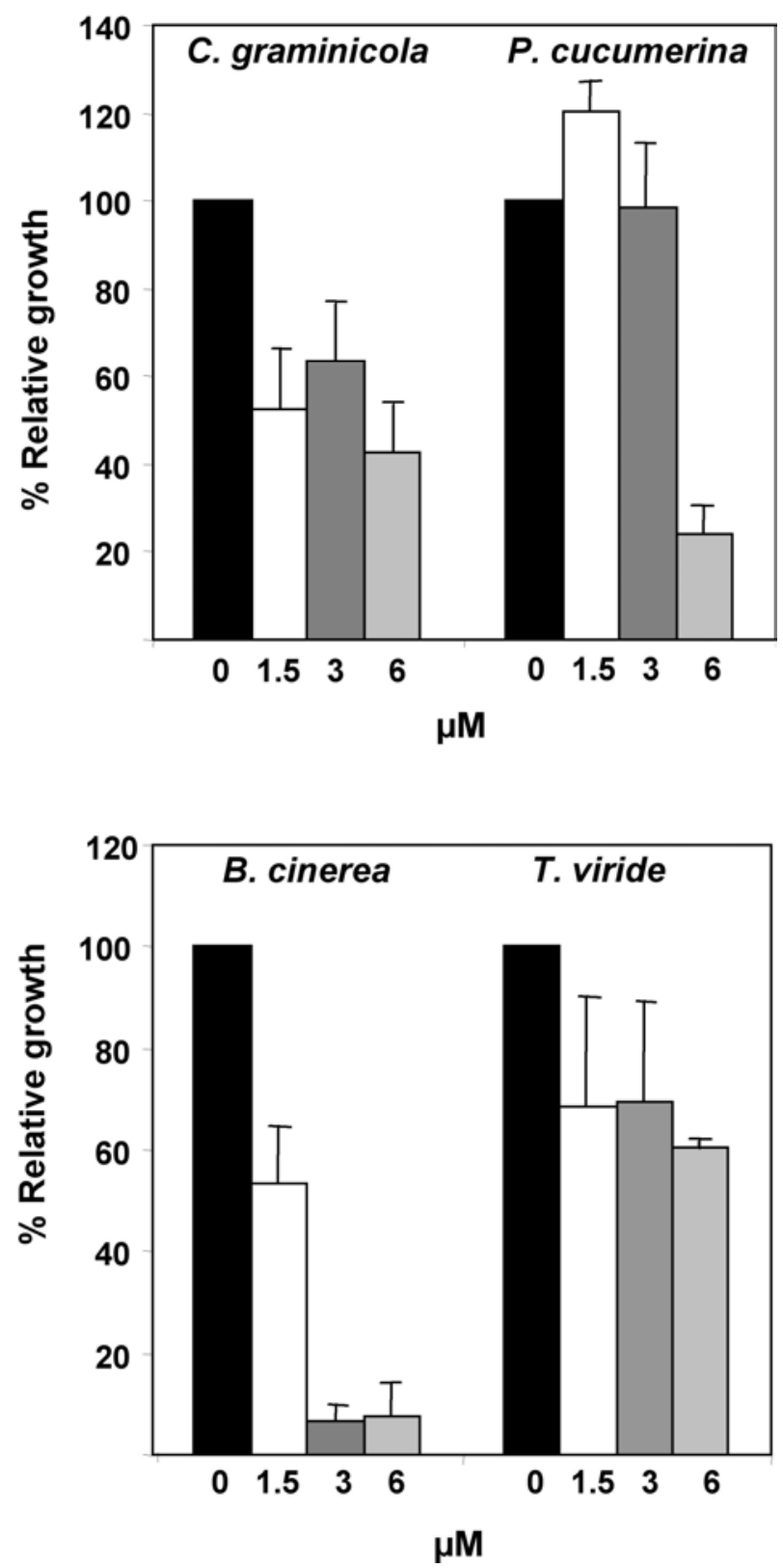

Fig. 1. Effect of the recombinant barley cystatin Hv-CPI on fungal growth. Several concentrations of the recombinant barley cystatin Hv-CPI were tested on the growth of Colletotrichum graminicola, Plectosphaerella cucumerina, Botrytis cinerea, and Trichoderma viride. Results are expressed as percentage of relative growth with respect to fungal growth in the absence of inhibitor. Data are mean values of four independent replicates. Bars indicate standard errors.

\section{RESULTS}

Inhibition of fungal growth

by recombinant barley $\mathrm{Hv}$-CPI.

Recombinant Hv-CPI, expressed in E. coli as a fusion protein of the complete open reading frame (ORF) (Gaddour et al. 2001) to a histidine tail, was purified by affinity chromatography with a $\mathrm{Ni}^{2+}$ column and used to study its effect on the growth of three phytopathogenic fungi, $C$. graminicola, $P$. cucumerina, and $B$. cinerea, and of one saprotrophic fungus, Trichoderma viride, by in vitro bioassays. The recombinant protein inhibited the growth of all the fungi tested (Fig. 1). For C. graminicola, effective concentration for $50 \%$ growth inhibition $\left(\mathrm{EC}_{50}\right)$ was obtained at 1.5 $\mu \mathrm{M}$ of Hv-CPI, while P. cucumerina was inhibited only at $6.0 \mu \mathrm{M}$. Growth was almost completely inhibited in $B$. cinerea at $3.0 \mu \mathrm{M}$. In vitro growth of the saprotrophic fungus $T$. viride decreased by $60 \%$ at the highest concentration tested. As expected, no growth inhibition was observed with protein purified from E. coli transformed with the expression vector without insert used as a negative control (data not shown). Thionin was included as a positive control because its antimicrobial properties are well documented (Garcia-Olmedo et al. 1999) and it was a strong inhibitor of all the fungi tested at a concentration of $6 \mu \mathrm{M}$ (data not shown).

The toxic effects that the recombinant Hv-CPI exerted on the growth of $B$. cinerea and $P$. cucumerina also were monitored by microscopic observations $24 \mathrm{~h}$ after the addition of the cystatin to the potato-dextrose broth (PDB) medium. Mycelial growth is strongly inhibited at $6.0 \mu \mathrm{M}$ (Fig. 2). In addition, morphological changes, such as hyphal shortening, were observed for $B$. cinerea.

\section{Production of barley cystatin variants by site-directed mutagenesis.}

Results in Figures 1 and 2 indicated that the barley cystatin had antifungal properties at the micromolar level, comparable to thionins, lipid transfer proteins, and other antimicrobial peptides (Garcia-Olmedo et al. 1999); therefore, we decided to generate cystatin mutants in order to study if any correlation was observed between their cysteine-proteinase inhibition and their fungicidal properties.

Using polymerase chain reaction (PCR) approaches (Table 1), seven cystatin mutants were produced by site-directed mutagenesis, with most mutations occurring in residues located in the conserved domains. Five mutants had point mutations with a single amino acid substitution in the Hv-CPI open read-

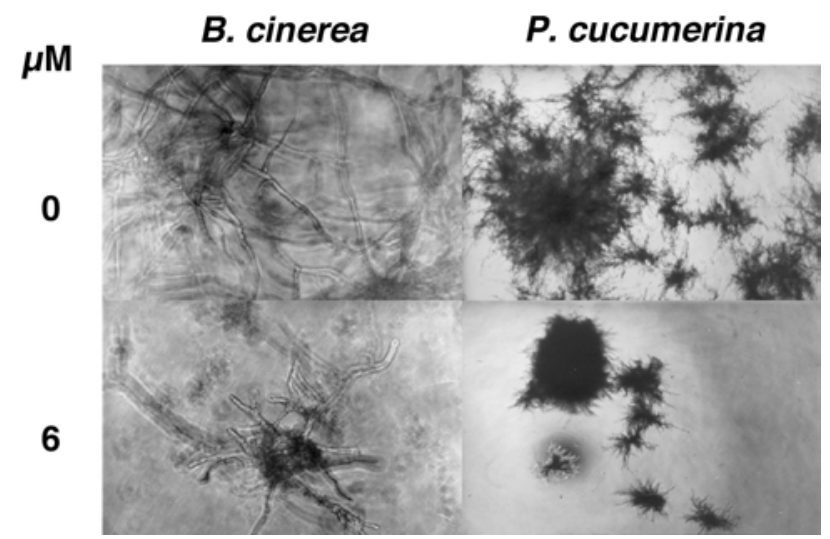

Fig. 2. Effect of the recombinant barley cystatin Hv-CPI on mycelial development. Growth arrest of Botrytis cinerea and Plectosphaerella cucumerina observed at the indicated concentrations of recombinant barley cystatin. Incubation was performed under the same conditions described for the fungal growth inhibition assays. Microscopic photographs (X300 for $B$. cinerea and X150 for $P$. cucumerina) were taken at $24 \mathrm{~h}$. 
ing frame: i) mutant $R^{38} \rightarrow G$ in the conserved cereal sequence ARFAV, ii) mutants $\mathrm{Q}^{63} \rightarrow \mathrm{L}$ and $\mathrm{Q}^{63} \rightarrow \mathrm{P}$ in the putative reactive domain (QVVAG), iii) mutant $\mathrm{C}^{68} \rightarrow \mathrm{G}$ in the unique cysteine residue, and iv) mutant $\mathrm{K}^{92} \rightarrow \mathrm{P}$, located contiguous to the $\mathrm{A}^{93} \mathrm{~W}^{94}$ motif near the $\mathrm{COOH}$ terminus. In addition, two truncated variants were generated: the mutant $\mathrm{Nterm}-\Delta \mathrm{Q}^{62}$, produced by the introduction of a stop codon instead of the glutamine in position $62\left(\mathrm{Q}^{62}\right)$ lacking the reactive domain QVVAG; and mutant $\Delta \mathrm{Q}^{62}$-Cterm, containing the last $\mathrm{C}$ terminal 45 residues that included the reactive site.

Although detailed structure-function studies were not the aim of this work, using a molecular model based on the known crystal structure of the rice OC-I (Nagata et al. 2000), which shares $79 \%$ sequence identity with the barley cystatin, the predicted structure of Hv-CPI was established (Fig. 3).
Barley Hv-CPI has an inferred three-dimensional structure similar to rice OC-I, containing an $\alpha$-helix where the ARFAV motif is located, and four of the five antiparallel $\beta$-sheets $\left(\beta_{2} \beta_{3} \beta_{4} \beta_{5}\right)$. The model predicts, instead of the OC-I $\beta_{1}$ sheet, situated $\mathrm{N}$-terminal to the $\alpha$-helix, two new small $\alpha$-helixes, located, at the $\mathrm{N}$-terminal and at the hairpin loop connecting the $\beta_{4}$ and $\beta_{5}$ sheets. The reactive site QVVAG is located at the end of the first $\beta$ sheet $\left(\beta_{2}\right)$ and in the hairpin loop connecting it to the next sheet $\left(\beta_{3}\right)$, (Fig. 3). Analyses of the models obtained for the barley cystatin point-mutants did not reveal any significant differences in their three-dimensional structures (data not shown), with the exception of mutant $\mathrm{Q}^{63} \rightarrow \mathrm{P}$, which lost the N-terminal small $\alpha$-helix and part of the first conserved $\beta$ sheet $\left(\beta_{2}\right)$; and mutant $\mathrm{K}^{92} \rightarrow \mathrm{P}$, which was devoid of the $\alpha$-helix located in the hairpin loop con-

Table 1. Oligonucleotide sequences of the primers used in the polymerase chain reaction experiments to generate barley cystatin variants ${ }^{\mathrm{a}}$

\begin{tabular}{|c|c|c|}
\hline Variants & Sequence & Primers \\
\hline \multirow[t]{2}{*}{ Hv-CPI (wt) } & 5'-GATCCATGGCCGAAGCGGCGCATG -3' & $\mathrm{P} 1$ \\
\hline & 5'-CAAAGCTTAGGCGGCCGGCTTGAA -3' & $\mathrm{P} 2$ \\
\hline \multirow[t]{2}{*}{ Mutant $\mathrm{R}^{38} \rightarrow \mathrm{G}$} & 5'-CGAGCTCGCGggcTTCGCCGTC-3' & P3 \\
\hline & 5'-GACGGCGAAgccCGCGAGCTCG-3' & $\mathrm{P} 4$ \\
\hline \multirow[t]{2}{*}{ Mutant $\mathrm{Q}^{63} \rightarrow \mathrm{L}$} & 5'-GTGAGGCAGctgGTGGTGGCCGG-3' & P5 \\
\hline & 5'-CCGGCCACCACcagCTGCCTCAC-3' & P6 \\
\hline \multirow[t]{2}{*}{ Mutant $\mathrm{Q}^{63} \rightarrow \mathrm{P}$} & 5'-GTGAGGCAGcegGTGGTGGCCGG-3' & P7 \\
\hline & 5'-CCGGCCACCACcggCTGCCTCAC-3' & P8 \\
\hline \multirow[t]{2}{*}{ Mutant $\mathrm{C}^{68} \rightarrow \mathrm{G}$} & 5'-GTGGCCGGGggcATGCACTAC-3' & P9 \\
\hline & 5'-GTAGTGCATgceCCCGGCCAC-3' & $\mathrm{P} 10$ \\
\hline \multirow[t]{2}{*}{ Mutant $\mathrm{K}^{92} \rightarrow \mathrm{P}$} & 5'-GGTGTGGGAGccgGCCTGGGAGA-3' & P11 \\
\hline & 5'-TCTCCCAGGCcggCTCCCACACC-3' & $\mathrm{P} 12$ \\
\hline \multirow[t]{2}{*}{ Mutant Nterm- $\Delta Q^{62}$} & 5'-GAAGGTGAGGtagCAGGTGGTG-3' & P13 \\
\hline & 5'-CACCACCTGctaCCTCACCTTC-3' & $\mathrm{P} 14$ \\
\hline Mutant $\Delta Q^{62}$-Cterm & 5'-GATCCATGGCAGCAGGTGGTGGCCGGGT-3' & P15 \\
\hline
\end{tabular}

${ }^{a}$ Restriction sites corresponding to HindIII (AAGCTT) and NcoI (CCATGG) are underlined and the ATG initiation codon is double underlined. The modified codons corresponding to the different mutants are in lower case and the base substitutions are indicated in bold.
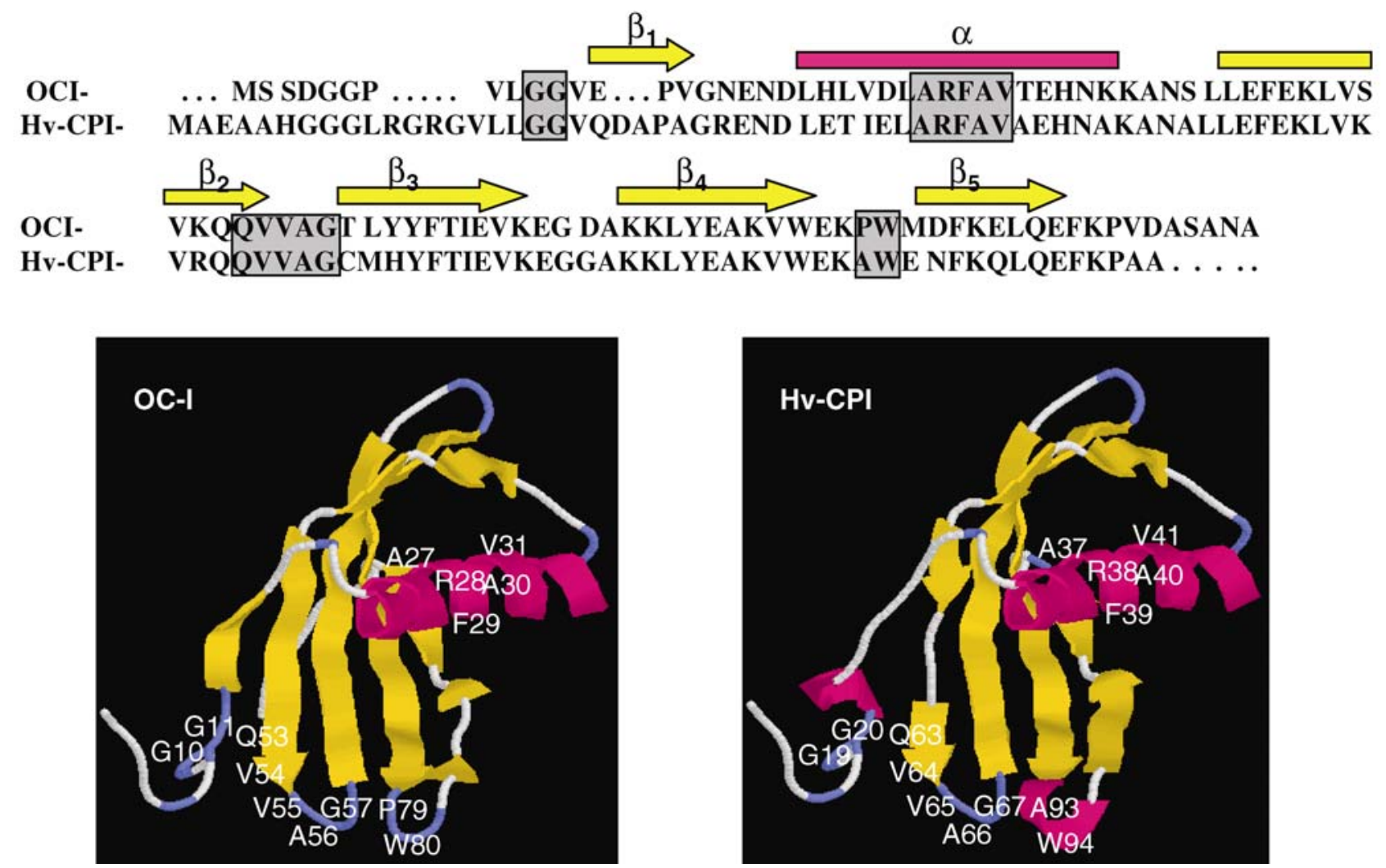

Fig. 3. Amino acid sequence alignment and ribbon plots of the oryzacystatin-I (OC-I) and the barley cystatin (Hv-CPI). The three-dimensional structure of Hv-CPI was predicted using the automated SWISS-MODEL program with the oryzacystatin I as a template. Figure prepared with RasMol 2.6. 
necting the $\beta_{4}$ and $\beta_{5}$ sheets, as well as part of these $\beta$ sheets themselves (data not shown).

\section{Variation of the $K i$ against papain and cathepsin $B$ of the Hv-CPI mutants.}

The recombinant Hv-CPI and its variants were expressed in $E$. coli as fusion proteins and assayed against papain and cathepsin B using $N$-benzoyl-DL-arginine- $\beta$-naphylamide (BANA) as substrate. Their $K \mathrm{i}$ values were determined (Table 2) as previously described (Gaddour et al. 2001). When the mutants $\mathrm{R}^{38} \rightarrow \mathrm{G}$ and $\mathrm{C}^{68} \rightarrow \mathrm{G}$ were assayed, their estimated $K$ i values for papain were $7.8 \times 10^{-5} \mathrm{M}$ and $1.2 \times 10^{-9}$ $\mathrm{M}$, and for cathepsin B were $5.9 \times 10^{-5} \mathrm{M}$ and $2.7 \times 10^{-8} \mathrm{M}$, respectively; while the $K$ i values of the wild type were $2.0 \times$ $10^{-8} \mathrm{M}$ for papain and $2.5 \times 10^{-6} \mathrm{M}$ for cathepsin $\mathrm{B}$. These data indicated that the $\mathrm{R}^{38}$ was an important amino acid for the inhibitory properties of this PhyCys, because its $K \mathrm{i}$ values clearly increased with respect to the wild type. However, the mutant $\mathrm{C}^{68} \rightarrow \mathrm{G}$ was a much better inhibitor than the wildtype $\mathrm{Hv}-\mathrm{CPI}$, because this substitution diminished its $K \mathrm{i}$ for papain by one order of magnitude and for cathepsin B by two orders of magnitude. On the other hand, mutants in the consensus region $\mathrm{Q}^{63}-\mathrm{V}^{64}-\mathrm{V}^{65}-\mathrm{A}^{66}-\mathrm{G}^{67}$ showed a drastic reduction of their activities against both cysteine-proteinases, as shown by the $K \mathrm{i}$ values for the mutant $\mathrm{Q}^{63} \rightarrow \mathrm{L}$ (150 and 50 times higher for papain and cathepsin $\mathrm{B}$, respectively, than that of the wild type) and for the mutant $\mathrm{Q}^{63} \rightarrow \mathrm{P}$, whose $K \mathrm{i}$ could not be detected. This decrease in inhibition activity also was observed in the mutant $\mathrm{K}^{92} \rightarrow \mathrm{P}$, with a $K$ i of $6.5 \times$ $10^{-4} \mathrm{M}$ for papain and $3.1 \times 10^{-4} \mathrm{M}$ for cathepsin $\mathrm{B}$ (four and two orders of magnitude, respectively, higher than the $K \mathrm{i}$ of the wild type). Finally, the two truncated variants, mutants Nterm- $\Delta Q^{62}$ and $\Delta Q^{62}$-Cterm, which lacked the C-terminal 46 residues and the $\mathrm{N}$-terminal 61 amino acids, respectively, showed no inhibitory activity (data not shown). Nevertheless, it is important to mention that, when the variant $\Delta \mathrm{Q}^{62}$-Cterm was expressed in E. coli, the corresponding recombinant protein accumulated in the inclusion bodies and its purification required denaturalization or renaturalization steps, which probably implied that the appropriate protein folding was not always achieved.

\section{Antifungal properties \\ of $\mathrm{Hv}-\mathrm{CPI}$ and its mutants against $\boldsymbol{B}$. cinerea.}

The phytopathogenic fungus $B$. cinerea was more susceptible to the antifungal activity of the wild-type Hv-CPI than the three other fungi tested (Figs. 1 and 2); therefore, we carried out in vitro bioassays of $B$. cinerea with the seven cystatin variants generated by mutagenesis. The assays were performed by adding increasing concentrations of each cystatin variant to the culture medium of $B$. cinerea and monitoring its effect on

Table 2. Inhibition constant $(\mathrm{Ki})$ values of barley cystatin variants against papain and cathepsin B and inhibition of the growth of Botrytis cinerea $^{\mathrm{a}}$

\begin{tabular}{lccc}
\hline & \multicolumn{2}{c}{ Ki $(\mathbf{M})$ against } & \\
\cline { 2 - 3 } Cystatin variants & Papain & Cathepsin B & $\mathbf{E C}_{\mathbf{5 0}}(\boldsymbol{\mu M})^{\mathbf{b}}$ \\
\hline Hv-CPI (wild type) & $2.0 \times 10^{-8}$ & $2.5 \times 10^{-6}$ & 1.66 \\
Mutant $\mathrm{R}^{38} \rightarrow \mathrm{G}$ & $7.8 \times 10^{-5}$ & $5.9 \times 10^{-5}$ & $2.49^{\mathrm{c}}$ \\
Mutant $\mathrm{Q}^{63} \rightarrow \mathrm{L}$ & $3.1 \times 10^{-6}$ & $1.4 \times 10^{-5}$ & $2.14^{\mathrm{c}}$ \\
Mutant $\mathrm{Q}^{63} \rightarrow \mathrm{P}$ & nd & nd & $2.10^{\mathrm{c}}$ \\
Mutant $\mathrm{C}^{68} \rightarrow \mathrm{G}$ & $1.2 \times 10^{-9}$ & $2.7 \times 10^{-8}$ & $2.37^{\mathrm{c}}$ \\
Mutant $\mathrm{K}^{92} \rightarrow \mathrm{P}$ & $6.5 \times 10^{-4}$ & $3.1 \times 10^{-4}$ & nd \\
\hline
\end{tabular}

${ }^{\mathrm{a}}$ No inhibitory activity detected $=$ nd.

${ }^{\mathrm{b}}$ Effective concentration for $50 \%$ inhibition $\left(\mathrm{EC}_{50}\right)$

${ }^{\mathrm{c}} \mathrm{EC}_{50}$ values were not statistically different $(P=0.05, t$ test $)$ fungal growth. After incubation for $48 \mathrm{~h}$, four of the five point mutants tested, with different $K \mathrm{i}$ values for papain and cathep$\sin \mathrm{B}$, produced an inhibition of fungal spore germination as effective as that produced by the recombinant $\mathrm{Hv}$-CPI itself (Fig. 4; Table 2). Only the point mutation $\mathrm{K}^{92} \rightarrow \mathrm{P}$ lacked fungicidal properties, although it still maintained a measurable inhibitory activity against papain and cathepsin B. This demonstrates that the lysine residue in position 92, although not essential for enzyme inhibition, was absolutely necessary for its antibiotic properties against fungi. According to the prediction of the structural model (data not shown), this mutant had lost the small $\alpha$-helix located in the hairpin loop connecting the $\beta_{4}$ and the $\beta_{5}$ sheets in the native Hv-CPI protein and part of the $\beta_{4}$ and $\beta_{5}$ sheets themselves.

The two truncated mutants, which lacked enzyme-inhibitory properties, did not affect fungal growth even at $6.0 \mu \mathrm{M}$, the highest concentration tested (data not shown). As expected, no antifungal properties were detected with the protein purified from $E$. coli transformed with the expression vector without insert used as negative control.

\section{Analysis of the proteinase activities of $B$. cinerea.}

To clarify the effect of PhyCys on the growth of $B$. cinerea, we checked for the presence of putative cysteine-proteinase activities in intra- and extracellular fungal extracts. Protein extracts from the mycelium and from the culture medium where $B$. cinerea had been grown were prepared and analyzed by mild denaturing gelatin-polyacrylamide gels, after incubation with different proteinase inhibitors. The addition of a range of commercial proteinase inhibitors with different classspecific proteinase inhibitory properties provided some information about the types of proteinase activities present in Botrytis spp. Gelatin-polyacrylamide electrophoretic gels showed several bands of hydrolytic activity (Fig. 5, lane C) that did not disappear after incubation with different specific inhibitors (Novillo et al. 1997), including the recombinant Hv-CPI, chicken egg white cystatin, E-64, iodoacetamide, and leupeptin (Fig. 5, lanes 2 through 6). This suggests the absence of cysteine-proteinase activities in $B$. cinerea under these conditions,

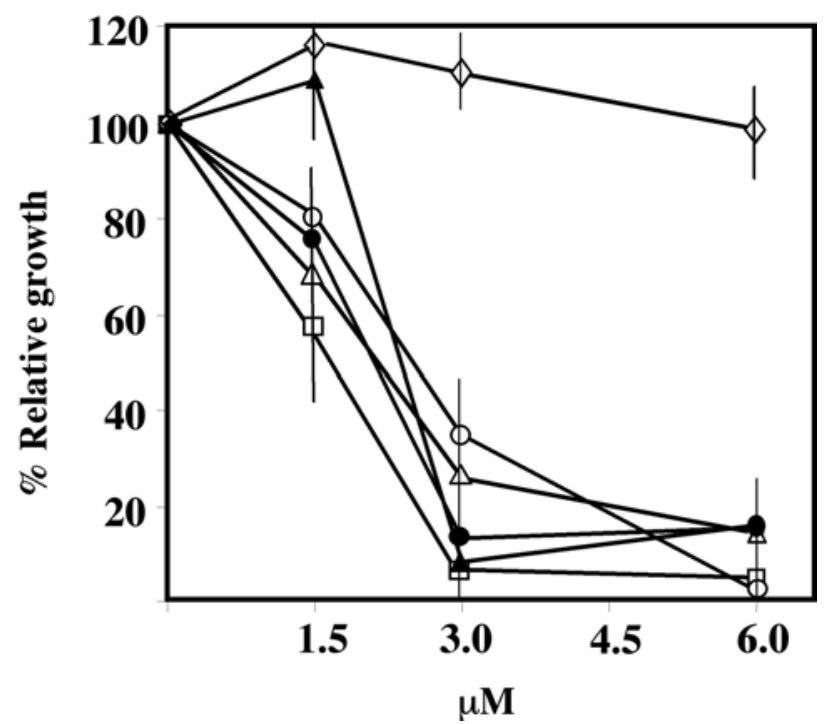

Fig. 4. Effect of the mutant variants derived from the Hv-CPI on the growth of Botrytis cinerea. Hv-CPI $(\square)$, mutant $\mathrm{R}^{38} \rightarrow \mathrm{G}(\mathrm{O})$, mutant $\mathrm{Q}^{63} \rightarrow \mathrm{L}(\Delta)$, mutant $\mathrm{Q}^{63} \rightarrow \mathrm{P}(\bullet)$, mutant $\mathrm{C}^{68} \rightarrow \mathrm{G}(\boldsymbol{\Delta})$, mutant $\mathrm{K}^{92} \rightarrow \mathrm{P}(\diamond)$. Results are expressed as percentage of relative growth versus fungal growth in the absence of inhibitor. Data are mean values of eight independent replicates. Statistical differences between data at $6.0 \mu \mathrm{M}$ were analyzed by the Student $t$ test. 
in which the $\mathrm{pH}$ used was the optimum to detect cysteineproteinases. The same results were obtained after incubation with pepstatin and EDTA, inhibitors of aspartyl- and metalloproteinases, respectively (Fig. 5, lanes 9 and 10). When the intraand extracellular protein fungal extracts were pre-incubated with the serine-proteinase inhibitor phenyl-methyl-sulfonylfluoride (PMSF), most of the bands in the zymograms disappeared (Fig. 5, lane 8), indicating that the majority of the observed protease activity corresponded to the serine-proteinase type. However, the Bowman-Birk trypsin inhibitor (BBI) from soybean had no appreciable effect on the intensity of the hydrolytic bands detected in these zymograms (Fig. 5, lane 7).

\section{DISCUSSION}

Plant proteinase inhibitors have been considered as natural defense phytochemicals against many species of herbivorous and pathogens. Their role as insecticidal proteins, attributed to their inhibitory activity toward digestive proteinases from insect pests and nematodes, has been established both by in vitro feeding assays and through transgenic expression in transformed plants (Kuroda et al. 1996; Pernas et al. 1998; Vain et al. 1998; Zhao et al. 1996). However, their antifungal properties and modes of action have been less explored. The antibiotic effect of the plant proteinase inhibitors against fungi was first described for serine-proteinase inhibitors (Lorito et al.

\section{Extra-cellular proteases}

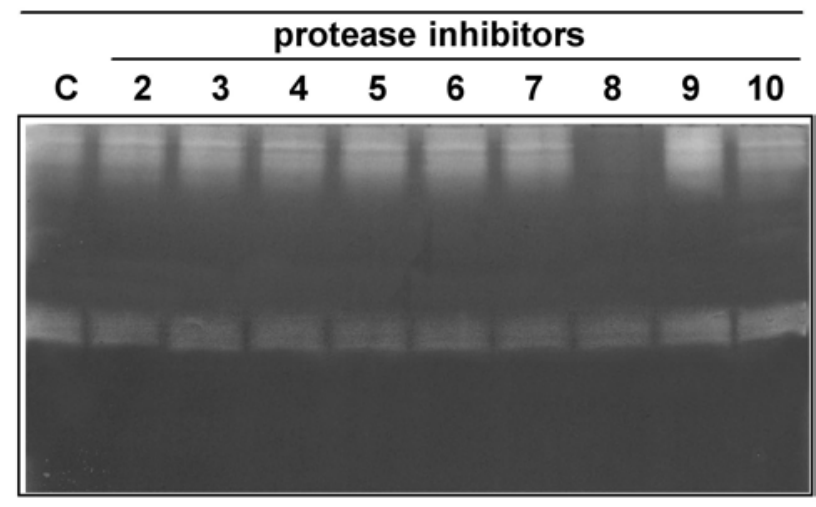

Intra-cellular proteases

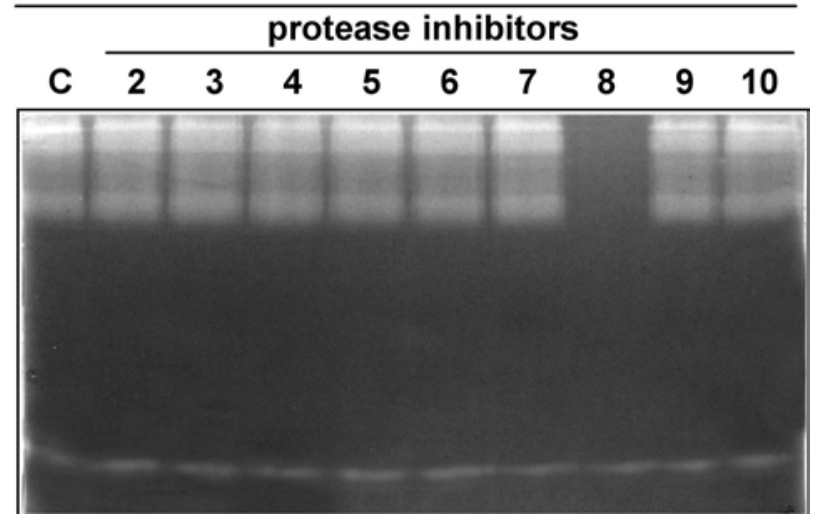

Fig. 5. Zymograms of the gelatinolytic activity of intra- and extracellular protein extracts from Botrytis cinerea and their inhibition. Detection of bands of hydrolytic activity after preincubation without proteinase inhibitors (C), or with $10 \mu \mathrm{M}$ recombinant barley cystatin (2), $1 \mu \mathrm{M}$ chicken egg white cystatin (3), $10 \mu \mathrm{M}$ E-64 (4), $10 \mathrm{mM}$ iodoacetamide (5), $100 \mu \mathrm{M}$ leupeptin (6), $1.5 \mu \mathrm{M}$ soybean Bowman Birk (7), $10 \mathrm{mM}$ phenyl-methyl-sulfonylfluoride (8), $100 \mu \mathrm{M}$ pepstatin (9), and $10 \mu \mathrm{M}$ EDTA (10).
1994; Terras et al. 1993) and, only more recently, reports of antifungal activity associated to PhyCys have started to appear (Joshi et al. 1999; Pernas et al. 1999; Siqueira-Junior et al. 2002; Soares-Costa et al. 2002).

We previously have reported the characterization of a cDNA from developing barley endosperm encoding the PhyCys HvCPI, and shown that it is a strong inhibitor of papain, chymopapain, and ficin in vitro (Gaddour et al. 2001). Now, we have demonstrated its potential in controlling the in vitro growth of three phytopathogenic and one saprotrophic fungi. The recombinant protein almost totally inhibited growth of $B$. cinerea at $3.0 \mu \mathrm{M}$, with an $\mathrm{EC}_{50}$ of $1.66 \mu \mathrm{M}$; higher concentrations were required to reduce the growth of $C$. graminicola, $P$. сuсumerina, and $T$. viride (Table 2 ). The potent inhibitory activity of Hv-CPI against $B$. cinerea was comparable (of the same order of magnitude) to that obtained when the antiphytopathogenic wheat thionin was added to the culture medium (GarciaOlmedo et al. 1999). The antifungal activity does not seem to be a general property of all the cysteine-proteinase inhibitors because chicken egg white cystatin and the synthetic inhibitor E-64 were not effective in parallel tests with the same fungal species (data not shown). The inability of both inhibitors to block fungal growth in vitro also was observed by Pernas and associates (1999) when Botrytis, Septoria, Colletotrichum, and Trichoderma spp. were assayed, and by Siqueira-Junior and associates (2002) after testing the effect of E-64 on Fusarium and Trichoderma spp. growth.

As a first approach to evaluate if the antifungal properties of $\mathrm{Hv}-\mathrm{CPI}$ were a consequence of its activity as a cysteine-proteinase inhibitor acting on fungal proteinases, systematic analysis of cysteine-proteinases inhibition and antifungal activity against $B$. cinerea was done for the recombinant Hv-CPI protein and all its mutant derivatives generated by PCR, as indicated in Table 1. Results in this study appear in Table 2. Three of the five point mutants obtained did not show significant differences in their three-dimensional structure compared with the wild type (data not shown). For two of them $\left(\mathrm{R}^{38} \rightarrow \mathrm{G}\right.$ and $\left.\mathrm{Q}^{63} \rightarrow \mathrm{L}\right)$, the estimated $K \mathrm{i}$ values for both cysteine-proteinases showed an important increase, indicating that $\mathrm{R}^{38}$ and $\mathrm{Q}^{63}$ were important amino acids for its inhibitory capacity, because their mutation substantially lowered their inhibitory activity (Table 2). Concerning the third mutant, the substitution of the only cysteine by a glycine $\left(\mathrm{C}^{68} \rightarrow \mathrm{G}\right)$ generated a greater cysteine-proteinase inhibitor, as shown by the $1.2 \times 10^{-9} \mathrm{M}$ and $2.7 \times 10^{-8} \mathrm{M}$ estimated antipapain and anticathepsin B Ki values, respectively, in comparison with the wild type. This result makes this mutant an excellent candidate to be used transgenically to improve crops against agronomically important insect and nematode pests (unpublished data). However, none of the residues modified in these variants seems to affect their antifungal activities, analyzed by in vitro bioassays against $B$. cinerea, which remained essentially similar to that of the recombinant Hv-CPI protein.

Two point mutation variants, $\mathrm{Q}^{63} \rightarrow \mathrm{P}$ and $\mathrm{K}^{92} \rightarrow \mathrm{P}$, in which the target amino acid residues were substituted by the cyclic amino acid proline, should have introduced important variations in the three-dimensional structure of the Hv-CPI mutant proteins. Surprisingly, the $\mathrm{Q}^{63} \rightarrow \mathrm{P}$ variant, which is devoid of cysteine-proteinase-inhibitory activity, was as good a fungicide against $B$. cinerea as the wild-type $\mathrm{Hv}-\mathrm{CPI}$. The $\mathrm{K}^{92} \rightarrow \mathrm{P}$ variant, which maintained significant, although reduced, antipapain and anticathepsin B activities compared with the wild-type, was inactive as fungicide, with no detectable effects either in spore germination or in growth of Botrytis mycelium. These results suggest that the antifungal activity of the barley cystatin Hv-CPI is not associated with its capacity to inhibit papain and cathepsin $\mathrm{B}$, and indicate that the sequence located close to 
amino acid $\mathrm{K}^{92}$ could span the antifungal domain. Finally, the Nterm- $\Delta Q^{62}$ variant, which lacks not only the sequence near the $\mathrm{K}^{92}$ residue but also the enzyme reactive site was, as expected, completely inactive both as a papain and cathepsin $\mathrm{B}$ inhibitor and as a fungicide. The $\Delta \mathrm{Q}^{62}$-Cterm variant, which maintained the reactive site and the putative antifungal domain, lacked both activities, probably indicating that sequences near the $\mathrm{N}$-terminal part of the molecule are important for maintaining the three-dimensional structure necessary both for protease inhibition and for its antifungal properties.

Taken together, all these data indicate that, in addition to the amino acids $\mathrm{Q}^{63}-\mathrm{V}^{64}-\mathrm{V}^{65}-\mathrm{A}^{66}-\mathrm{G}^{67}$, corresponding to the consensus region of the cysteine-proteinase reactive site and the $\mathrm{K}^{92}$ $\mathrm{P}^{93}-\mathrm{W}^{94}$ domain, the sequence common to cereal cystatins $\mathrm{A}^{37}$ $\mathrm{R}^{38}-\mathrm{F}^{39}-\mathrm{A}^{40}-\mathrm{V}^{41}$ and the $\mathrm{C}^{68}$ residue are important for barley cystatin activity. Moreover, the $\mathrm{K}^{92}$ residue or the three-dimensional organization of the residues located around it are probably important for the antifungal effect. Alvarez-Fernandez and associates (1999) have described a cystatin C from mammals able to inhibit papain and legumain with two different nonoverlapping reactive sites involved in the two different activities.

Although the mechanism of fungal inhibition by Hv-CPI is not fully understood, this activity does not seem not to be mediated by cysteine-proteinase inhibition. This also is suggested by the lack of detection of intra- and extracellular cysteineproteinase activities in $B$. cinerea zymograms, under experimental conditions where serine-proteinases could be detected. The production and secretion of trypsin-like proteinases have been described in other pathogenic fungi (Girard and Michaud 2002; Pekkarinen and Jones 2002). Pernas and associates (1998) reported that the chestnut cystatin not only had an anticysteine-proteinase activity but also had an in vitro inhibitory activity against trypsin, a well known serine-proteinase. This antitrypsin activity could not be detected for Hv-CPI (data not shown).

It has been speculated that the antifungal properties of some PhyCys could be related to the inhibition of fungal cysteine proteinases involved in the activation of chitin synthase. Although this matter merits further investigation, it is pertinent to mention that, using the threading techniques of sequence-to-structure alignments (LooppV2.0 program Muller Elber R, Cornell Theory Center), we found local structural similarities to a lectin (1JacA). Lectins are carbohydrate binding proteins which have been associated with hostpathogen interactions, such as a lectin from Phaseolus vulgaris, that can suppress the growth of several fungal species (Ye et al. 2001).

In conclusion, our data suggest that different protein domains in the barley cystatin, Hv-CPI, are associated with its antifungal and its cysteine-proteinase inhibitory properties. It is also important to consider that one of the cystatin variants $\left(\mathrm{C}^{68} \rightarrow \mathrm{G}\right)$ generated in this study is a good candidate as an improved transgene for obtaining a better insect control, while retaining good antipathogenic properties, both of which are interesting aspects for the biotechnological improvement of crops in the future.

\section{MATERIAL AND METHODS}

\section{Fungal growth inhibition assays.}

Fungal strains from the laboratory collection (B. cinerea, $T$. viride, $C$. graminicola, and $P$. cucumerina) were grown in potato-dextrose agar (Difco Laboratories, Detroit) medium at $28^{\circ} \mathrm{C}$. The in vitro inhibition assays were performed as described by Pernas and associates (1999). Approximately $10^{4}$ spores of each fungal strain were incubated in $100 \mu \mathrm{l}$ of onethird PDB (Difco Laboratories) at $28^{\circ} \mathrm{C}$ for $48 \mathrm{~h}$ in the presence of measurable concentrations of the recombinant barley cystatin or of its derived mutants. The incubation was carried out in sterile microtiter plates and fungal growth was monitored by measuring absorbance at $492 \mathrm{~nm}$.

\section{Bacterial expression and purification \\ of the recombinant barley cystatin protein (Hv-CPI) and its mutant forms from $E$. coli.}

The complete ORF of the barley cystatin cDNA (Gaddour et al. 2001) was amplified by PCR using P1 as the forward primer, which incorporated a NcoI site in frame with the ATG initiation codon; and P2 as the reverse primer, which added a HindIII site in its $3^{\prime}$ end (Table 1). The amplified DNA fragment was inserted into the fusion expression vector pRSETB (Invitrogen, San Diego, CA, U.S.A.) and introduced into $E$. coli BL21 (DE3) pLysS. Bacterial cells over-expressing barley cystatin were harvested after $2 \mathrm{~h}$ of induction with IPTG (isopropyl $\beta$-D-thiogalactopyranoside) (Sigma, Madrid). The fusion protein with the N-terminal histidine tag was purified to homogeneity, using a His-Band Resin and elution conditions from the $\mathrm{Ni}^{+}$-column following manufacturer's instructions (Novagen, Madison, WI, U.S.A.).

Five point mutants of the barley cystatin cDNA were obtained using site-directed mutagenesis by a recombinant PCR approach. In the first PCR round, a single base change was introduced in two overlapping cDNA fragments using the primers derived from the $5^{\prime}$ and $3^{\prime}$ ends of the Hv-CPI cDNA (Table 1, P1 and P2), combined independently with overlapping internal primers which contained the base substitutions (Table 1, P3 to P12). In the second round, the two fragments were annealed and extended using the P1 and P2 primers described above. The base substitutions introduced, which were checked by DNA sequencing, produced the following amino acid alterations in the wild-type Hv-CPI protein: $\mathrm{R}^{38} \rightarrow \mathrm{G}$, $\mathrm{Q}^{63} \rightarrow \mathrm{L}, \mathrm{Q}^{63} \rightarrow \mathrm{P}, \mathrm{C}^{68} \rightarrow \mathrm{G}$, and $\mathrm{K}^{92} \rightarrow \mathrm{P}$. In addition, two truncated variants were generated. The Nterm- $\Delta Q^{62}$ mutant, containing the first $\mathrm{N}$-terminal 61 amino acid residues of the HvCPI, was obtained by the recombinant PCR approach previously described, using as the overlapping internal primers P13 and P14 (Table 1), which changed the $\operatorname{Gln}^{62}$ to a stop codon. The $\Delta \mathrm{Q}^{62}$-Cterm mutant, which included the last 45 C-terminal residues preceded by ATG initiation codon, was generated by a PCR with the primers P15 and P2 (Table 1). All the resultant barley cystatin variants were digested with NcoI and HindIII and inserted in frame into the fusion vector pRSETB (Invitrogen), after which the recombinant proteins were purified to homogeneity as described above. Their electrophoretic mobilities in sodium dodecyl sulfate (SDS) polyacrylamide gel electrophoresis were consistent with the changes introduced (data not shown).

\section{Structural models.}

The three-dimensional structure of mutants and wild-type barley cystatins were modeled using the automated SWISSMODEL program (Peitsch 1995,1996). The known crystal structure of the rice OC-I (PDB identifier 1EQK) was used to construct the homology-based models. Structure analysis was performed using RasMol 2.6 program (Sayle and Milner-White 1995).

\section{Cysteine-proteinase inhibitory activity.}

Inhibitory activity of the recombinant cystatin Hv-CPI and its derived protein mutants was tested against papain (EC 3.4.22.2) and cathepsin B (EC 3.4.22.1) essentially as described by Gaddour and associates (2001), using BANA (Merck, Barcelona, Spain) as substrate. Protein concentrations were quantified by the BioRad kit with bovine albumin as standard and $K$ i values determined from Dixon plots (1/V versus [I]). 


\section{Proteolytic activity in mycelium and extracellular protein extracts from $B$. cinerea.}

To analyze the extra- and intracellular protease activities of $B$. cinerea, $500 \mathrm{ml}$ of $1 / 3 \times$ PDB (Difco Laboratories) were inoculated with $10^{6}$ spores and incubated at $28^{\circ} \mathrm{C}$. After 1 week, mycelium was pelleted by centrifugation at $5,000 \mathrm{rpm}$ for $15 \mathrm{~min}$ and the extracellular medium was concentrated by lyophilization by a factor of approximately 30 . The mycelium was freeze dried and ground, and the resulting powder defatted with ethanol:ether $(1: 3, \mathrm{vol} / \mathrm{vol})$ for $1 \mathrm{~h}$. After drying, the soluble proteins were extracted with water $(1: 8$, $\mathrm{wt} / \mathrm{vol})$ and the mycelial debris pelleted by centrifugation $\left(10,000 \mathrm{rpm}\right.$ for $30 \mathrm{~min}$ at $\left.4^{\circ} \mathrm{C}\right)$.

Proteolytic activity of protein extracts from $B$. cinerea was electrophoretically analyzed using mild denaturing gelatine or polyacrylamide gels according to the procedure described by Michaud (1998). Protein (15 $\mu \mathrm{g})$ from the mycelium extracts and $1 \mu \mathrm{g}$ of fungal extracellular protein were incubated at room temperature for $10 \mathrm{~min}$ with the following proteinase inhibitors: $10 \mu \mathrm{M}$ recombinant barley cystatin Hv-CPI, $1 \mu \mathrm{M}$ chicken egg white cystatin (Sigma), $10 \mu \mathrm{M}$ E-64 (L-transepoxysuccinyl-leucylamido-[4-guanidino] butane; Merck), $10 \mathrm{mM}$ iodoacetamide (Sigma), $100 \mu \mathrm{M}$ leupeptin (Merck), $10 \mathrm{mM}$ PMSF (Merck), $1.5 \mu \mathrm{M}$ soybean BBI (Merck), 100 $\mu \mathrm{M}$ pepstatin (Merck), and $10 \mu \mathrm{M}$ EDTA (Sigma). The reaction mixtures were electrophoresed in $0.1 \%$ (wt/vol) SDS$12 \%$ (wt/vol) polyacrylamide gels containing $0.05 \%(\mathrm{wt} / \mathrm{vol})$ gelatine at $4{ }^{\circ} \mathrm{C}$; the gels were transferred to a $2.5 \%(\mathrm{vol} / \mathrm{vol})$ aqueous solution of Triton X-100 for $45 \mathrm{~min}$, then incubated in $1 / 3 \times$ PDB (Difco Laboratories) containing $5 \mathrm{mM}$ cysteine (Sigma) at $37^{\circ} \mathrm{C}$ for $3 \mathrm{~h}$ and, finally, stained as described by Michaud (1998).

\section{ACKNOWLEDGMENTS}

We thank M. Milgroom (Cornell University, Ithaca, NY) for the critical reading of the manuscript. Financial support from the Comunidad Autónoma de Madrid-Spain (projects 07M/0015/2001 and 07M/0050/2002) and from the Ministry of Science and Technology-Spain (project P98-0734) are gratefully acknowledged. M. Martínez was the recipient of a Ramón y Cajal contract from Ministerio de Ciencia y Tecnología (Spain).

\section{LITERATURE CITED}

Abe, M., Domoto, C., Watanabe, H., Abe, K., and Arai, S. 1996. Structural organization of the gene encoding corn cystatin. Biosci. Biotechnol. Biochem. 60:1173-1175.

Alvarez-Fernandez, M., Barrett, A., Gerhartz, B., Dando, P. M., Ni, J., and Abrahamson, M. 1999. Inhibition of mammalian legumain by some cystatins is due to a novel second reactive site. J. Biol. Chem. 274:19195-19203.

Arai, S., Watanabe, H., Kondo, H., Emori, Y., and Abe, K. 1991. Papain-inhibitory activity of oryzacystatin, a rice seed cysteine proteinase inhibitor, depends on the central Gln-Val-Val-Ala-Gly region conserved among cystatin superfamily members. J. Biochem. 109:294-298.

Bateman, A., Birney, E., Cerrutti, L., Burbin, R., Etwiller, L., Eddy, S. R., Griffiths-Jones, S., Howe, K.-L., Marshall, M., and Sonnhammer, E. L. 2002. The Pfam protein families database. Nucleic Acids Res. 30:276280.

Corre-Menguy, F., Cejudo, F. J., Mazubert, C., Vidal, J., LelandaisBriere, C., Torres, G., Rode, A., and Hartmann, C. 2002. Characterization of the expression of a wheat cystatin gene during caryopsis development. Plant Mol. Biol. 50:687-698.

Delledonne, M., Allegro, G., Belenghi B., Balestrazzi, A., Picco, F., Levine, A., Zelasco, S., Calligari P., and Confalonieri, M. 2001. Transformation of white poplar (Populus alba L.) with a novel Arabidopsis thaliana cysteine proteinase inhibitor and analysis of insect pest resistance. Mol. Breed. 7:35-42.

Gaddour, K., Vicente-Carbajosa, J., Lara, P., Isabel-Lamoneda, I., Diaz, I., and Carbonero, P. 2001. A constitutive cystatin-encoding gene from barley (Icy) responds differentially to abiotic stimuli. Plant Mol. Biol. 45:599-608.

Garcia-Olmedo, F., Molina, A., Alamillo, J. M., and RodríguezPalenzuela, P. 1999. Plant defence peptides. Biopolymers 47:479-491.

Girard, C., and Michaud, D. 2002. Direct monitoring of extracellular protease activities in microbial cultures. Anal. Biochem. 308:38-391.

Gutierrez-Campos, R., Torres-Acosta, J., Saucedo-Arias, L. J., and Gomez-Lim, M. A. 1999. The use of cysteine proteinase inhibitors to engineer resistance against potyviruses in transgenic tobacco plants. Nature Biotechnol. 17:1223-1226.

Joshi, B. N., Sainani, M. N., Bastawade, K. B., Gupta, V. S., and Ranjekar, P. K. 1998. Cysteine protease inhibitor from pearl millet: a new class of antifungal protein. Biochem. Biophys. Res. Commun. 246:382-287.

Joshi, B. N., Sainani, M. N., Bastawade, K. B., Deshpande, V. V., Gupta, V. S., and Ranjekar, P. K. 1999. Pearl millet cysteine protease inhibitor. Evidence for the presence of two distinct sites responsible for anti-fungal and anti-feedant activities. Eur. J. Biochem. 265:556-563.

Kondo, H., Abe, K., Nishimura, I., Watanabe, H., Emori, Y., and Arai, S. 1990. Two distinct cystatins in rice with different specificities against proteinases. J. Biol. Chem. 265:15832-15837.

Kuroda, M., Ishimoto, M., Suzuki, K., Kondo, H., Abe, K., Kitamura, K., and Arai S. 1996. Oryzacystatins exhibit growth inhibitory and lethal effects on different species of bean insect pests. Biosci. Biotechnol. Biochem. 60:209-212.

Lorito, M., Broadway, R. M., Hayes, C. K., Woo, S. L., Noviello, C., Williams, D. L., and Harman, G. E. 1994. Proteinase inhibitors from plants as a novel class of fungicides. Mol. Plant-Microbe Interact. 7:525-527.

Margis, R., Reis, E. M., and Villeret, V. 1998. Structural and phylogenetic relationship among plant and animal cystatins. Arch. Biochem. Biophys. 359:24-30.

Michaud, D. 1998. Gel electrophoresis of proteolytic enzymes. Anal. Chem. Acta 372:173-185.

Nagata, K., Kudo, N., Abe, K., Arai, S., and Tanokura M. 2000. Threedimensional solution of oryzacystatin-I, a cysteine proteinase inhibitor of the rice, Oryza sativa L. japonica. Biochemistry 39:14753-14760.

Novillo, C., Castañera, P., and Ortego, F. 1997. Characterization and distribution of chymotrypsin-like and other digestive proteases in Colorado potato beetle larvae. Arch. Insect Biochem. Physiol. 36:181-201.

Peitsch, M. C. 1995. Protein modeling by E-mail. Biotechnology 13:658660.

Peitsch, M. C. 1996. ProMod and Swiss-Model: Internet-based tools for automated comparative protein modeling. Biochem. Soc. Trans. 224:274-279.

Pekkarinen, A. I., and Jones B. L. 2002. Trypsin-like proteinases produced by Fusarium culmorum grown on grain proteins. J. Agric. Food Chem. 19:3849-3855.

Pernas, M., López-Solanilla, E., Sanchez-Monge, R., Salcedo, G., and Rodriguez-Palenzuela, P. 1999. Antifungal activity of a plant cystatin. Mol. Plant-Microbe Interact. 12:624-627.

Pernas, M., Sanchez-Monge, R., Gomez, L., and Salcedo, G. 1998. A chestnut seed cystatin differentially effective against cysteine proteinase inhibitor from closely related pests. Plant Mol. Biol. 38:1235-1242.

Pernas, M., Sanchez-Monge, R., and Salcedo, G. 2000a. Biotic and abiotic stress can induce cystatin expression in chestnut. FEBS (Fed. Eur. Biochem. Soc.) Lett. 467:206-210.

Pernas, M., Sánchez-Ramos, I., Sanchez-Monge, R., Lombardero, M., Arteaga, C., Castañera, P., and Salcedo, G. 2000b. Der p1 and Der $\mathrm{f} 1$, the highly related and major allergens from house mites, are differentially affected by a plant cystatin. Clin. Exp. Allergy 30:972978.

Sayle, R., and Milner-White, E. J. 1995. RasMol: Biomolecular graphics for all. Trends Biochem. Sci. 20:374.

Siqueira-Junior, C. L., Fernandes, K. V. S., Machado, O. L. T., Cunha, M., Gomes, V. M., Moura, D., and Jacinto, T. 2002. 87 kDa tomato cystatin exhibits properties of a defence protein and forms crystals in prosytemin over-expressing transgenic plants. Plant Physiol. Biochem. 40:247-254.

Soares-Costa, A., Beltramini, L. M., Thiemann, O. H., and Enrique-Silva, F. 2002. A sugarcane cystatin: recombinant expression, purification and antifungal activity. Biochem. Biophys. Res. Commun. 296:1194-1199.

Terras, F. R. G., Schoofs, H. M. E., Thevissen, K., Osborn, R. W. Vanderleyden, J., Cammue, B. P. A., and Broekaert, W. F. 1993. Synergistic enhancement of the antifungal activity of wheat and barley thionins by radish and oilseed rape $2 \mathrm{~S}$ albumins and barley trypsin inhibitors. Plant Physiol. 103:1311-1319. 
Vain, P., Worland B, Clarke, M. C., Richard, G., Beavis, M., Liu, H., Kholi, A., Leech, M., Snape, J., Christou, P., and Atkinson, H. R. 1998. Expression of an engineered cysteine proteinase inhibitor (Oryzacystatin-I $\triangle \mathrm{D} 86)$ for nematode resistance in transgenic rice plants. Theor. Appl. Genet. 96:26-271.

Walker, A. J., Urwin, P. E., Atkinson, H. J., Brain, P., Glen, D. M., and Shewry, P. R. 1999. Transgenic Arabidopsis leaf tissue expressing a modified oryzacystatin shows resistance to the field Deroceras retuculatum (Mull). Transgenic Res. 8:95-103.
Ye, X. J., Ng, T. B., Tsang, P. W., and Wang, J. 2001. Isolation of a homodimeric lectin with antifungal and antiviral activities from red kidney bean (Phaseolus vulgaris) seeds. J. Protein Chem. 20:367375.

Zhao, Y., Botella, M. A., Subramanian, L., Niu, X., Nielsen, S. S., Bressan, R. A., and Hasegawa, P. M. 1996. Two wound-inducible soybean cysteine proteinases have greater insect digestive proteinase inhibitory activities than a constitutive homolog. Plant Physiol. 111:1299-1306. 